\title{
Surgical treatment of heart failure
}

\author{
Marina Alves Jacintho de Mello*, Camila Alcalde Mazza and Edmo Atique Gabriel \\ Union of the Colleges of the Great Lakes- UNILAGO, Brazil
}

\begin{abstract}
The general objective of this study is to make a survey of the scientific production about the surgical treatment for Chronic Heart Failure (CHF). As a methodology, an integrative review was used to collect scientific productions. The research was carried out through a survey of the published journals in electronic databases: Latin American Literature in Health Sciences (LALIHS), Scientific Electronic Library Online (SCIELO) and Portal of the Ministry of Health, considering the articles inherent to the objective here traced. It is believed that the awareness of people can reduce the number of hospitalizations and deaths that involve $\mathrm{CHF}$ in Brazil, not excluding secondary prevention and pharmacological treatment to prolong the life of the patient already affected by the problem, however, health education may be the best way to reduce these numbers. With surgical treatment being only indicated in cases of hospital emergency associated with the need for revascularization in the ischemic myocardium, ventricular reconstruction and correction of secondary mitral insufficiency.
\end{abstract}

\section{Introduction}

Chronic Heart Failure (CHF) is the focus of this study, which aims, through a bibliographic review, to make a survey about the surgical treatment for this disease.

It is understood that Heart Failure has been one of the major reasons for death of adults in the country, there are no epidemiological studies that demonstrate the real problem that involves the disease, thus, it is believed that study aimed at the identification of prevalence and mortality of the CHF are fundamental, considering that many cases can be avoided from primary prevention actions. Thus, it is assumed that the citizens' awareness about the disease can reduce the morbidity and mortality rates due to CHF in the country.

In this context, the study is justified by its social and professional relevance. It is socially relevant because it can bring CHF to the knowledge of citizens as a very common problem, sometimes unknown by the population though; and professionally relevant because according to prevalence and mortality survey for this disease health professionals may have it as a basis for future actions.

The general objective of this study is to make a survey of the scientific production about the surgical treatment for CHF. And the specific objectives in: make a study on the Brazilian guidelines for the care of patients with CHF; and to identify the forms of treatment for CHF.

As a methodology, an integrative review was used to collect scientific productions. The research was carried out through a survey of the published journals in electronic databases: Latin American Literature in Health Sciences (LILACS), Scientific Electronic Library Online (SCIELO) and Portal of the Ministry of Health, considering the articles inherent to the objective here traced.

\section{Chronic heart failure: concepts and definitions}

Chronic Heart Failure (CHF) is characterized by a cardiac dysfunction, where the heart cannot pump enough blood to meet the need for oxygen and tissue nutrition, a complex clinical syndrome that leads to a reduction in blood flow elevation of pulmonary and systemic venous pressures $[1,2]$.

Vidal (2013) [3] stated that heart failure is characterized by a series of multifactorial phenomena, which may compromise both ventricular relaxation and the body's metabolic needs. In turn, Expósito et al. [4] points out that $\mathrm{CHF}$ is interpreted as a syndrome that produces peripheral organ malfunction in relation to its metabolic demand, due to processes that affect, in a primary or secondary way, the capacity of the heart to function properly.

According to Prompt et al. [5], it is a chronic and progressive disease, affecting mainly adults over 65 years. Barreto and Ramires [6] add that the population aging in recent years has been increasing the incidence of CHF. On the subject, Moreira and Omura [2] say: Heart failure (HF) is a complex clinical syndrome, classically defined as the inability of the heart to pump adequate blood supply, in relation to venous return and tissue metabolic needs, causing reduction of cardiac output and elevation of pulmonary and systemic venous pressures. Although many believe that myocardial infarction is the leading cause of death from heart problems, the champion of mortality is heart failure. In fact, according to the World Health Organization, there has been an increase in the incidence of Heart Failure (HF) in the world, making it the most current clinical challenge in public health, and is still considered a growing epidemic problem. In Brazil, it is estimated that 6.4 million Brazilians suffer from HF. It is also believed that the increase in the number of elderly people in the population, predicted by World Health Organization, should result in the multiplication of the cases by the disease.

Thus, it is possible to say that $\mathrm{CHF}$ is a public health problem. The high incidence and lethality caused by this disease is a constant stage

*Correspondence to: Marina Alves Jacintho de Mello, Avenue José Munia, 6300- São José do Rio Preto/SP- Brazil; Tel: 5517997884321; E-mail: mazzacamila5@hotmail.com

Key words: heart failure, public health, surgical treatment

Received: December 05, 2018; Accepted: December 18, 2018; Published: December 21, 2018 
of studies, according to Kamel et al. [7], this is because even with the diversity of treatments used, the high incidence rate and morbimortality remain, in addition to a significant impairment of the quality of life, so the more one knows about this dysfunction, the greater the chances to prolong the patient's life and even prevent its occurrence.

The etiology of CHF is quite broad and may involve any pathology leading to cardiac morphofunctional changes, among them coronary artery disease, hypertension, degenerative valvular disease and atrial fibrillation [8]. Summarizing the etiology of CHF, Oliveira [9] states: In summary, there are a large number of diseases and alterations that lead to the development of HF but can be classified into three groups regarding the etiology: 1 . Ventricular contractility disorders. Ex: Chagas disease and acute myocardial infarction; 2. Pressure and volume overload. Ex: arterial hypertension and valvular diseases; 3. Ventricular filling disorders. Ex: ventricular hypertrophies. Recent studies indicate that angiotensin II causes vascular wall: increased intracellular calcium, DNA and protein synthesis, hypertrophy of smooth muscle cells, increased expression of pre-pro-endothelin from smooth muscle cells and endothelial cells, and, indirectly, increased release of norepinephrine in the presynaptic junctions.

Expósito et al. [4] divided CHF etiology into CHF systolic and Diastolic CHF, as shown in Table 1.

Concerning to clinical manifestations, CHF has signs and symptoms directed at the heart's inability to pump blood, as well as to transport the necessary nutrients to the human body. According to Candia [10], its symptoms are progressive, and may appear when the individual is active, however, over time other respiratory problems begin to affect the individual even when resting. It should be noted that although the symptoms are progressive, CHF can also appear suddenly after a heart attack or other heart problem. Among the most common symptoms of CHF, the author cites:

Shortness of breath in physical activity or shortly after lying down for a while; Cough; Swelling of the feet and ankles; Swelling of the abdomen; Weight gain; Irregular or rapid pulse; Feeling of feeling the heart beat (palpitations); Difficulty sleeping; Fatigue, weakness, fainting; Loss of appetite, indigestion; Decreased attention or concentration; Reduced urine volume; Nausea and vomiting; Need to urinate at night; Babies may have sweat during feeding (or other activity).

It is important to emphasize that the signs and symptoms presented by the individual will depend on the condition to which his heart is submitted, as well as which of the ventricles is most involved, for example, the left ventricle usually fails faster, but both failures may occur at the same time.

When the left ventricle goes into insufficiency, it congests the pulmonary territory and causes a progressive lack of air that can even manifest itself at rest. It is called orthopnea - the condition in which the

Table 1. CHF etiology
\begin{tabular}{|c|c|}
\hline Systolic CHF & Diastolic CHF \\
\hline Myocardial ischemia & Hypertension tension \\
\hline Arterial hypertension & Aortic stenosis \\
\hline Diabetes Mellitus & Hypertrophic cardiomyopathy \\
\hline Toxic (Including Alcohol) & Restrictive myocardial diseases \\
\hline Infections (Endocarditis) & Restrictive pericardial diseases \\
\hline Heart valvular lesions & Myocardial ischemia \\
\hline Dilated cardiomyopathy & \\
\hline Cardiac arrhythmias & \\
\hline Idiopathic & \\
\hline
\end{tabular}

person feels short of breath even when lying down, which forces him to wake up at night to settle (paroxysmal nocturnal dyspnea). This left insufficiency may develop into an even more severe condition, called acute pulmonary edema, which can end in death if not treated urgently. Other symptoms, less severe, but very common are: shortness of breath, tachycardia, dyspnea, nocturnal cough, etc. In right ventricular failure the right ventricle cannot pump enough blood into the lung. As such, the fluids revert to the veins and capillaries and accumulate in the tissues causing edema (swelling), perceived mainly in the legs, because of the force of gravity. (ABC.MED.BR, 2011 p.1) [11].

Thus, CHF symptoms are generally directed at respiratory failure, especially when the individual is active, such as in the practice of a physical exercise, and may vary the symptoms according to the affected ventricle.

\section{Guidelines for chf patient care}

The guidelines for patient care with CHF are described based on the publication of Brazilian Archives of Cardiology written by Bocchi et al. [12], which brings the update of Brazilian guideline for Chronic Heart Failure 2012.

Initially, there is a search for diagnosing the disease, with reference to electrocardiogram, troponin and polymorphism, BNP / NTproBNP, Ecodopplercardiogram, Nuclear Medicine - SPECT and PET, Cardiac Computed Tomography and Magnetic Resonance and Ergospirometric Test.

Considering that $\mathrm{CHF}$ is a public health problem, it was believed that the approach of these patients should be made from a standard, in order to facilitate the medical action in identifying the needs of each patient.

It is worth noting that family evaluation, as well as other approaches with the patient, is of great importance, due to familial character of some HF etiologies; it is necessary the evaluation and the family screening should any suspicion of these come up.

The treatment prescribed to patient by doctors is also a point that needs a lot of attention: as previously highlighted, it may be pharmacological and non-pharmacological, or a combination of both; and it is fundamental that the initial approach provides subsidies to health care professional, so that he can check the best treatment according to the specific case of each patient.

In view of the above, it can be said that the CHF is a complex problem, which has been shown to be of great incidence in Brazil, presenting high morbidity and mortality rates, and its treatment is carried out considering each specific case.

\section{Treatment}

Generally, the treatment performed for CHF is aimed to relieving patient's symptoms, as well as improving their quality of life and survival, while seeking to prevent disease progression, using pharmacological and non-pharmacological means for treatment [13].

Brunner and Suddarth [14] elucidate that the CHF treatment aims to eliminate or reduce any etiological factors that contribute to its progression, such as atrial fibrillation and excessive alcohol intake, and also aims at reducing the workload over the heart by decreasing afterload and preload. Thus, the treatment carried out involves the modification of patients' lifestyle, to water restriction, hyposodic diet, oxygen therapy and pharmacological therapy. 
It is important to emphasize that CHF treatment should be done considering the individualized factors of each patient, according to Cândia [10]. The treatment prescribed will depend on factors such as the cause of heart failure, symptoms and the possible presentation of clinical complications. Besides, the disease stage also needs to be considered.

Santos [15] complements that age should also be taken into account, since it brings about the occurrence of metabolism alterations and the normal drug excretion imposed by it, not to mention that elderly are affected by other diseases, also considered comorbidities. In addition, the author also mentions the fact that the elderly are more likely to present adverse reactions due to ingestion of treatment drugs.

Ferraz and Omura [16] emphasize the need for a multidisciplinary approach in the treatment of CHF, stating that a large team of trained and specialized professionals is needed for its treatment.

On the subject, Joaquim et al. [13] state: At all stages of HF, nonpharmacological measures correspond to the basis of treatment, without which control of risk factors and the use of medications to control symptoms tend to become ineffective and the patient progresses in his disease. Under this context, the multidisciplinary approach, with the active participation of professionals from the areas of Nutrition, Hospital Pharmacy, Physiotherapy, Psychology, Social Care and Occupational Therapy, should be considered essential for success in the clinical management of all HF patients. Therefore, guidelines on the most appropriate diet, correct use of medications, regular physical and respiratory exercises, and leisure activities, as well as an appropriate approach to psychosocial disorders, including the involvement of all close family members, should be the core of the adherence to the chronic treatment of his disease. Without this, even the use of stateof-the-art and high-tech drugs can become obsolete and ineffective for patients.

In this context, the treatment of CHF needs a range of professionals, as well as the involvement of the family, so that the patient's adherence to treatment can be facilitated, considering it to be complex since it involves a change of life habits.

Expósito et al. [4] argue that CHF treatment can be done through primary and secondary prevention, in addition to being generalized or specific.

Primary prevention involves the actions to be performed before the disease is affected with the individual avoiding the risk factors that cause the different types of heart diseases. While secondary prevention is performed after the disease is affected, soon after diagnosis and throughout life, changes are made in the patient's lifestyle in order to prevent the progression of the disease [4].

As for the generalized treatment for all patients affected by CHF, there is sodium restriction, water restriction, physical exercise, and health education for the patient, from food education to correct adherence to treatment [4].

Finally, the specific treatment is explained by [4] as the one should consider the specificities of each patient, involving pharmacological and non-pharmacological treatment.

Non-pharmacological treatment of CHF, according to Ferraz and Omura [16] involves the search for clinical stabilization and improvement of functional capacity. Functional capacity is understood as the capacity of the individual to perform tasks of daily life, to interact socially, in addition to exercising cognitive functions, his emotional state and the resulting economic condition.

Regarding the non-pharmacological treatment for CHF, Joaquim et al. [13] elucidate: Among the non-pharmacological measures established, the salt-restricted diet is of vital importance for the reduction of water retention, which is one of the main characteristics of the HF patient. The intensity of the sodium restriction may depend on the stage of the disease, but generally ranges from 1 to $2 \mathrm{~g}$ of salt per meal. Hypertensive patients will already benefit from the hyposodic diet offered under HF conditions, and those with chronic renal failure may require even greater salt restriction. Regarding water restriction, it is recommended to ingestion of liquids at a maximum of 1000 to 1500 $\mathrm{mL} /$ day for those patients with significant weight gain and who are hyponatremic (serum sodium below $130 \mathrm{mEq} / \mathrm{L}$ ) and / or refractory to use of diuretics. In addition, medical consensus has guided the daily measurement of the patient's body weight at the household level in order to allow the use of safer and smaller doses of diuretics, in addition to reducing the frequency of exacerbations of congestive symptoms and the number of hospital emergencies.

Thus, the non-pharmacological treatment of CHF involves changes in patients' life habits, from salt and water consumption, to the search for adequate nutrition and physical exercise.

Ferraz and Omura [16] elucidate that the non-pharmacological treatment requires the joint action of different health professionals, nurses, physiotherapists, physicists, nutritionists, psychologists, social workers and physicians, acting in a multidisciplinary way, guidance and education.

Chiesa, Fracoli and Nogueira [17] define as health education: "any combination of learning experiences with a view to facilitating voluntary actions conducive to health". In addition, the authors point out that such actions may assume more conservative or libertarian perspectives, therefore, not every project with this focus would be emancipatory in its own right.

Regarding health education as a strategy for the treatment of CHF, Ferraz and Omura [16] highlight: These strategies aim to transmit informative content, strengthen medical prescription, clarify doubts, alleviatedistress, discussgoals and eventual treatment failures. The patient should be encouraged to look for incipient signs of decompensation by means of home measurements of weight, ankle circumference, and circumference of the abdomen, as well as worsening of symptoms such as dyspnea and orthopnea. Alcohol use should be discouraged in patients with HF. During prolonged travel they should receive special attention due to the risk of dehydration, edema accentuation and the occurrence of deep venous thrombosis. Wandering and movement of the lower limbs, and the use of elastic stockings are recommended. Aerobic physical exercises are indicated with cyclic movements and involving large muscle groups. Examples include walking, cycling and slow running. Annual influenza vaccination is recommended and every three years against pneumonia due to respiratory infection, which can trigger the decompensation of the heart disease, aggravating the ventricular dysfunction. Regarding diets, patients should be advised on sodium restriction and water restriction. They should also be guided regarding the effects of medications on survival and quality of life, names and doses of medications, probable side effects, intake and regular schedules.

Thus, diet and physical exercises are fundamental factors for the treatment of CHF, being highlighted by Joaquim et al. [13] that the sedentary lifestyle is responsible for the worsening of the quality 
Table 2. Surgical treatments heart failure

Revascularization in the ischemic myocardium

Ventricular Reconstruction

Mitral valve surgery
Ischemic myocardial revascularization Myocardial ischemic dysfunction may be reversed by surgical treatment, significantly contrasting with isolated clinical management, as demonstrated by several studies, constituting a major criterion for operative indication.

The impact of resection of dyskinetic areas of the left ventricle in the evolution of ischemic heart disease has long been recognized. Well-conducted experimental studies support the concept of surgically avoiding the expansion of infarcted areas with impairment of the remaining myocardial function. It is therefore advisable, in view of these clinical-experimental evidences, that in the indication and planning of the surgical treatment of patients with ischemic cardiomyopathy and fraction of reduced ejection the presence of areas of fibrosis should be studied preferably by means of cardiac magnetic resonance and regional motility tagging.

This regurgitation is based on the left ventricle sphericity, which will determine the papillary spacing, ring dilatation and additional loss of contractile function, with increased wall tension and increased energy expenditure. This technique has as a principle to correct additional overload to the left ventricle, eliminating the regurgitant flow and increasing the systolic volume without having primarily the pretension to improve the ejection fraction. of life of the patients, as well as the worsening of the prognosis. In this context, the non-pharmacological treatment of CHF is aimed at improving the patients' quality of life and preventing the progression of the disease.

Pharmacological treatment, as well as non-pharmacological treatment, must consider patients in their peculiarities, from the history of the disease, to their personal characteristics, so that they can achieve success.

According to Oliveira [9], the purpose of pharmacological treatment is to increase the cardiac output so that it is possible to reduce the symptoms of CHF and prolong the patient's life.

Ferraz and Omura [16] complement that they affirm that for decades the pharmacological treatment for CHF was based only on digital and diuretics, however with the advance of the knowledge about the disease, new therapeutic approaches have been used, aiming to act on the congestive and as well as in neurohormonal activation, endothelial dysfunction and cardiac remodeling, and it should be emphasized that angiotensin-converting enzyme (ACE) inhibitors and beta-blockers are mandatory.

Boundy [18] elucidates that CHF can generally be treated with a combination of drugs involving: Diuretics (such as furosemide, hydrochlorothiazide, spironolactone, ethacrynic acid, bumetanide or triamterene) to reduce total blood volume and circulatory congestion; Inotropic agents such as digoxin to increase cardiac contractility; Sympathomimetics such as dopamine and dobutamine in acute situations; or amrinone to increase contractility and cause arterial vasodilation; Vasodilators to increase cardiac output or angiotensin converting enzyme (ACE) inhibitors to reduce afterload; Several drugs are also being investigated in the treatment of heart failure; Betaadrenergic blockers to improve myocardial response.

Finally, It should be emphasized that the medicines must be prescribed by the doctor according to the peculiarities of each patient, since it consists of a specific treatment, and its efficiency is based on each specific case.

With regard to surgical treatment, it is possible to verify the revascularization in the ischemic myocardium, the ventricular reconstruction and the correction of the secondary mitral insufficiency, which are described in Table 2.

\section{Conclusion}

The CHF was seen in this study as a public health problem, responsible for a high percentage of hospitalizations and deaths in Brazil, even though, in most cases, it can be prevented by changing life habits and behaviors, it continues to affect thousands of Brazilians.

It is believed that the awareness of people can reduce the number of hospitalizations and deaths that involve CHF in Brazil, not excluding secondary prevention and pharmacological treatment to prolong the life of the patient already affected by the problem, however, health education may be the best way to reduce these numbers. With surgical treatment being only indicated in cases of hospital emergency associated with the need for revascularization in the ischemic myocardium, ventricular reconstruction and correction of secondary mitral insufficiency.

\section{References}

1. Smeltzer SC, Bare, BG Brunner, Suddarth (2006) Medical-Surgical Nursing Treaty. 10th ed. Rio de Janeiro: Guanabara Koogan.

2. Ferraz AS, Bocchi EA, Guimarães GV, Meneghelo RS, Moreira DAR, et al. (2003) Effects of Aerobic Exercise Training on Autonomic Balance, Brain Natriuretic Peptide, and Quality of Life Evaluation in Dilated Cardiomyopathy Patients. A Prospective Randomized Controlled Study. Circulation 108: 739.

3. Vidal TMS, Brandão SCS, Brandão DC, Batista GR (2013) Interactive aerobic exercise in the rehabilitation of patients with heart failure: a systematic review of the literature. ABCS Health Sci 38: 166-171.

4. Negrin EJE, Cordiés JL, Roselló SN, Sanchez RJ, Negrin VJA (2001) Chronic heart failure. Revista Cubana.

5. Alberto PC, Rafael de A, Nadine C, Andréa B, Fábio B(2009) Peritoneal dialysis (PD) as a treatment for congestive heart failure (CHF) in patients with chronic stage IV renal disease. J Bras Nefrol 31: 220-222.

6. Pereira BAC, Ramires José Antonio Franchini (1998) Heart Failure. Arq Bras Cardiol.

7. Kamel Cesar Selem, Barreto Luiz Felipe Mena, Benchimol Marcos, Siqueira-Filho Aristarco G (2001) Congestive Heart Failure. Correlation between the functional class and the systolic and diastolic functions evaluated by the Doppler ultrasound. Arq Bras Cardiol.

8. (2016) Proceedings of the 3rd IPLeiria's International Health Congress: Leiria Portugal. 6-7 May 2016. BMC Health Serv Res 16 Suppl 3: 200. [Crossref]

9. Oliveira, Edilberto Antonio Souza de Oliveira (2015) Drugs Used in the Treatment of Congestive Heart Failure (CHF).

10. Maggioni AP, Anker SD, Dahlström U, Filippatos G, Ponikowski P, et al. (2013) Are hospitalized or ambulatory patients with heart failure treated in accordance with European Society of Cardiology guidelines? Evidence from 12,440 patients of the ESC Heart Failure Long-Term Registry. Eur J Heart Fail 15: 1173-1184. [Crossref]

11. ABC.MED.BR, 2011 p. 1

12. Bocchi EA, Marcondes-braga FG, Bacal F, Ferraz AS, Albuquerque D, et al. (2012) Brazilian Society of Cardiology. Update of the Brazilian Guideline for Chronic Heart Failure - 2012. Arq Bras Cardiol 98: 1-33.

13. Joaquim LF, Volpe GJ, Figueiredo AB, Moriguti JC (2010) Pipes in the patient with heart failure hospitalized in internal medicine wards. Medicine (Ribeirão Preto) 43: 93-106.

14. Buffolo E, White JNR, Catani R, RESTORE Group (2006) End-stage cardiomyopathy and secondary mitral insufficiency - Surgical alternative with prostheses implantation and left ventricular restoration. Eur J Cardiothorac Surg 29: S266-S271.

15. Santos Eduarda Barcellos dos, Villacorta Bolívar Saenz Tello Humberto, Sales Ana Luisa Ferreira, Wiefels Christiane, Teixeira Pedro Soares (2010) Anemia and Heart Failure in the Community: Comparison with a Specialized Outpatient Clinic. Arq Bras Cardiol 94: 102-108.

16. Ferraz Renata da Silva, Omura Carina Michel (2005) Nursing performance in the treatment of Heart Failure. Journal of Nursing UNISA 6: 80-84. 
17. Chiesa Anna Maria, Fracolli Lislaine Aparecida, Nogueira Vanessa Franquini. The promotion of health and health education as a field of knowledge and practices.
18. Boundy Janice (2004) Medical-surgical nursing. 3rd ed, Vol. 2, Reichmann \& Affonso and Editores, 583-587.

Copyright: $\odot 2018$ Jacintho de Mello MA. This is an open-access article distributed under the terms of the Creative Commons Attribution License, which permits unrestricted use, distribution, and reproduction in any medium, provided the original author and source are credited. 